\title{
ADVANTAGE OF A GREEN AND SMART PORT OF THE FUTURE
}

\author{
JOAO PITA COSTA ${ }^{1}$, IGNACIO LACALLE ${ }^{2}$, MIGUEL A. LLORENTE ${ }^{3}$, OLIVIER LE BRUN ${ }^{4}$, \\ LEONIDAS PTSIKAS ${ }^{5}$, GILDA DE MARCO ${ }^{6}$, CHARLES GARNIER ${ }^{7}$, ERWAN SIMON ${ }^{7}$, \\ ALEX GHERGHINA ${ }^{8}$, ORESTIS TSOLAKIS ${ }^{9}$, DEJAN ŠTEPEC ${ }^{1}$, SERGIO VIVO ${ }^{3}$, RAFAEL VAÑO ${ }^{2}$, \\ STJEPAN PILIČIĆ ${ }^{10}$, TEODORA MILOŠEVIĆ ${ }^{10}$, MATIJA ŠIROKA ${ }^{10}$, FABRICE KLEIN $^{11}$, \\ STEFANO BEVILACQUA ${ }^{12}$, TAMARA CORSANO ${ }^{13}$, CINZIA NINZATTI ${ }^{13}$, \\ ATHANASIOS CHALDEAKIS ${ }^{14}$, GRIGORIS DIMITRIADIS ${ }^{15}$, LUKA TRAVEN $^{10}$, \\ FLAVIO FUART ${ }^{1} \&$ CARLOS E. PALAU ${ }^{2}$ \\ ${ }^{1}$ XLAB Razvoj Programske Opreme In Svetovanjel (XLAB), Slovenia \\ ${ }^{2}$ Dpto. De Comunicaciones, Universitat Politècnica de València (UPV), Spain \\ ${ }^{3}$ Prodevelop, Spain \\ ${ }^{4}$ CREOCEAN, France \\ ${ }^{5}$ PEOPLE, UK \\ ${ }^{6}$ Informatica Per Il Sistema Degli Enti Locali (INSIEL), Italy \\ ${ }^{7}$ Centre Aquitain Des Technologies de l'Information et Electroniques (CATIE), France \\ ${ }^{8}$ ORANGE, France \\ ${ }^{9}$ Ethniko Kentro Erevnas Kai Technologikis Anaptyxis (CERTH), Greece \\ ${ }^{10}$ Sveučilište U Rijeci, Medicinski Fakultet (MEDRI), Croatia \\ ${ }^{11}$ Grand Port Maritime du Bordeaux (GPMB), France \\ ${ }^{12}$ Autorità di Sistema Portuale del Mare Adriatico Orientale (AdSP MAO), Italy \\ ${ }^{13}$ Stazioni Doganali Autoportuali Gorizia (SDAG), Italy \\ ${ }^{14}$ Piraeus Port Authority (PPA), Greece \\ ${ }^{15}$ Thessaloniki Port Authority (THPA), Greece
}

\begin{abstract}
The digitalization is undeniably arriving to the port industry. However, modern digital technologies had not pervaded before in the sector because of, mainly, ports' complexity and heterogeneity as wide ecosystems. When it comes to applying innovative digital tools in maritime ports, a series of additional common barriers are usually faced: (i) unavailability of clear reference in open-source based technologies, (ii) closed-environments and high pricing rates of private providers, (iii) restricting regulations inside and outside the ports preventing port authorities to deploy useful products and (iv) high heterogeneity of objectives, data or perspectives to carry out focused accountable actions. The PIXEL project has helped ports of all sizes to overcome those barriers during the last three years. Throughout a variety of domains of action - including clean energy, environmental performance, smart intermodal transport or machine learning-based maritime data analytics, PIXEL has addressed those hindrances, driving four European ports towards the Port of The Future stand. The different open applications developed form an ecosystem that may be adopted by external ports aiming at improving their digitalisation levels and their operational and environmental performance. For the 2021 edition, we are presenting the suite of tools deployed in the ports in the context of the action, their success stories and best practices, and how they can be leveraged by worldwide maritime transport entities in the future. It is our objective to provide a comprehensive review of their functioning, technical traits and particularities and how they are planned to be exploited by the Consortium. The PIXEL team truly believes that this will mean a milestone in the operational research field for the ports sector. Finally, we aim at offering a perspective on the usage of modern technologies in maritime ports based on the experience and lessons learned in the conduction of PIXEL project and the interaction with other initiatives in the period 2018-2021.
\end{abstract}

Keywords: maritime industry, internet of things, Big Data, environmental performance port operations. 


\section{INTRODUCTION}

For a while now the maritime industry has been taking small steps towards the digitalisation of overall operations and actions towards better energy efficiency and environmental performance [1] for an efficient global response to the climate crisis in an era of IoT-based digital twins arriving to the port [2] deriving from the work done in Industry 4.0 [3].

The equilibrium between cost saving and performance optimization can be hard to tackle when civil societies, local governments and maritime agencies are more aware and demand efficiency. PIXEL is the first smart, flexible and scalable solution for reducing environmental impacts while enabling the optimization of operations in port ecosystems through the Internet of Things (IoT) [4]. It enables collaboration of ports, multimodal transport agents and cities for optimal use of internal and external resources, sustainable economic growth and environmental impact mitigation, towards the Ports of the Future. It allows for the reduction of environmental impact of port activities, an increase of renewable energy uptake in usecases at small, medium and large ports, and a reduction of operational and infrastructural costs with better Port-City integration.

The flagship tool of PIXEL is an environmental index driven by the data collected by the IoT infrastructure at the port. It allows to quantify, in a single composite indicator, the impact that one port is having with regards to environmental interaction with its surroundings. Strongly rooting on IoT techniques and heterogeneous data, becoming a useful tool for analysing the evolution of port authorities, terminals and vessels' environmental impacts in time. However, PIXEL is more than an environmental index. It is an innovative, robust and secure IoT platform that can optimise port operations while evaluating the environmental performance of the port based on the same data collected through plugged-in FIWARE [5] agents. It enables modelling a simulation of energy, pollution and COVID-19 restrictions' impact in a terminal supply chain, but it allows also to include new models that can be useful. Furthermore, it offers powerful predictive algorithms to forecast and better plan vessel traffic as well as to optimise intermodal transport through the city and at the port gates [6].

In this paper we present the PIXEL innovation and its operational and environmental value, made easy and accessible to ports of all sizes, with interest beyond the port community (e.g., shipping agents, Smart Cities within local governments, or maritime authorities and other agencies). We describe the components of the platform in its core functionalities (PEI, PAS and Predictive Algorithms) as well as its technological enablers (Data Acquisition Agents, Information Hub, Model Manager and Customisable Dashboard), as can be seen in Fig. 1. The technology was validated on the needs of small and medium but also large ports, ranging from the Port of Monfalcone (recently merged with the Port of Trieste) to the Port of Piraeus, the largest in Europe moving goods.

The main aim of this paper (and its primary contribution to the state of the art) is the description of a novel 3-dimensional modularity of an IoT system (see Fig. 2) - over (i) computer models adopted; (ii) components that can be substituted by existing ICT infrastructure (e.g., the security model); and (iii) the data acquisition agents, allowing for the plug-in of different data sources - ensures that the digitalisation of the port can better fit the different rhythms for that transition.

\section{PORT ENVIRONMENTAL INDEX}

Addressing the current concerns in the context of the climate crisis, transversal to all industries, and in line with the European Green Deal [7], PIXEL is proposing an IoT-driven environmental index tailor fit to ports of all sizes. 


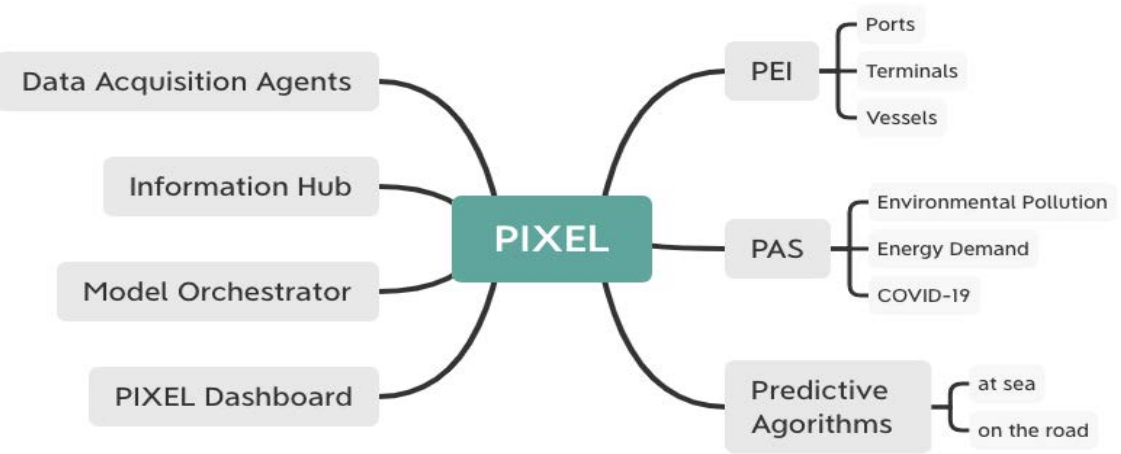

Figure 1: The building blocks of the PIXEL Ports technology.

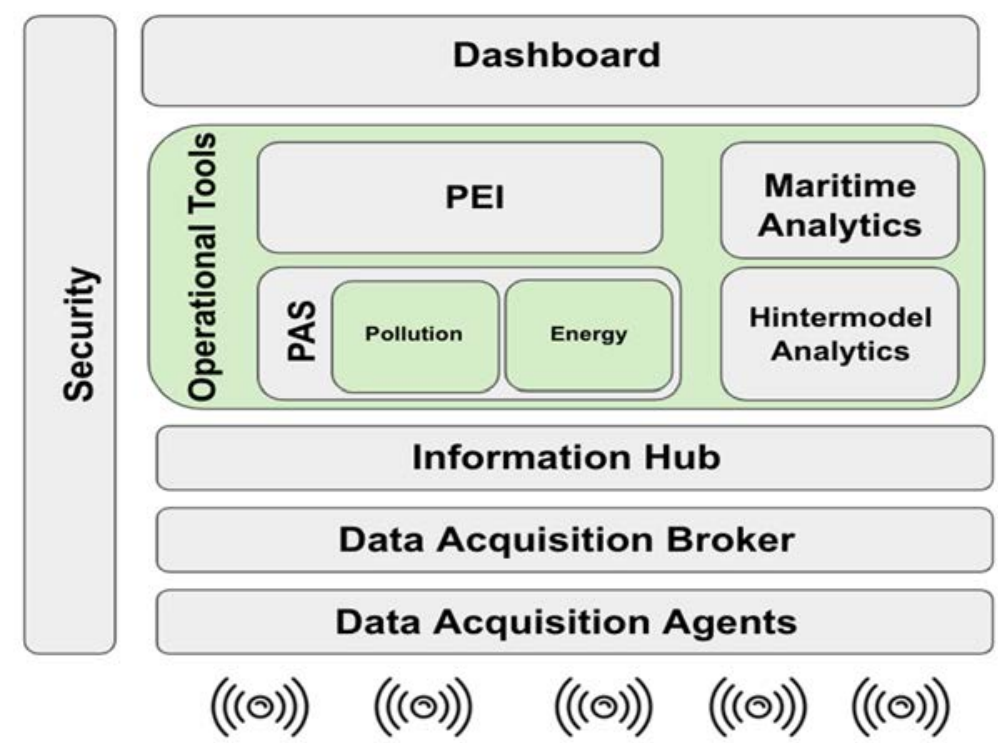

Figure 2: The different dimensions of PIXEL's modularity.

\subsection{Towards the port of the future}

Over the years, ports have become increasingly aware of the importance of their role, not only in global supply chains but also in society, an important aspect of which includes the environmental impact of their activities [8]. This is why an increasing number of ports are implementing environmental metrics to monitor the impact of their activities in areas such as air and water quality, waste generation, noise and light emissions as well as resource consumption (e.g., energy or water resources [9]). In the context of the PIXEL project, a detailed recording of these metrics was performed, through the content analysis of the published environmental reports by TEN-T ports [10] along with relevant information collected through an online, structured questionnaire survey. The result of this work [9] 
showed that a large variety of metrics is currently used by the European ports, customised according to their needs in an attempt to assess their environmental behaviour. However, it appears that despite these consistent efforts, in the majority of cases ports are lacking the ability to have an overall assessment of their environmental behaviour, mainly due to the lack of a single metric framework which will integrate all the relevant environmental aspects of port operations. To fill this gap, in the context of the PIXEL project, a single composite metric called Port Environmental Index (PEI) was developed, in order to be used for selfmonitoring, appraisal of different mitigation measures as well as for reporting issues (interport comparisons, and benchmarking against best practices). This metric was designed to have minimum requirements to be operational, enabling the vast majority of European to implement it regardless of their size. Its scalability and portability have been validated in PIXEL through use-cases and a transversal trial. The adoption of PEI is vital to address the Port of the Future paradigm [11] in a conscious, actionable and valuable relationship with the local community and biodiversity. There are several angles to this paradigm shift, including the efforts in the use of renewable energies (also addressed by PIXEL, see Section 3.2) or the adoption of circular economies, and there are some approaches to evaluating environmental performance [12], [13], but none is data-driven, European-wide and considers the limited resources of small and medium ports at the level that PIXEL does.

\subsection{Quantifying the environmental performance at the port}

The PEI is a global quantitative environmental index fed by real-time data, allowing ports to assess the progress of their own environmental performance. The method and the related index enable flexibility and scalability to monitor key environmental indicators in real-time through the IoT infrastructure at the port [14]. By using PEI, ports can obtain a real-time measure of their environmental footprint and to plan actions to mitigate adverse effects. The environmental performance is segmented by different categories of emissions and pollutants and can be monitored per category and per type of contributing entity. PEI is split into several sub-indices depending on the type of impact (air pollution, waste and wastewater, light, noise and odour) and origin of the pollution source. The measurements are valid across ports of all sizes and data infrastructure capabilities, allowing to increment accuracy with better data input. PEI allows port authorities to optimise the use of resources at the port and appropriately monitor environmental-related activity in order to act upon it. Enhancement of environmental performance planning capabilities to improve the green image of the port and attract new customers are also valuable traits associated with this tool. This scientific novelty [14] is addressing the complex problem of quantifying the environmental performance of the port terminal - vessel in real-time and at a low cost, viable for small and medium ports. It is based on a quantitative composite indicator of the overall environmental performance of a port, from data collected by the minimal IoT infrastructure at the port. It allows all sized ports to measure their environmental performance and to compare with each other regardless of their size. PIXEL collects environmental data from heterogeneous sources and combines them into a single quantitative composite indicator. Using the complex algorithms developed, the collected data is homogenised and used to calculate the composite indicator which quantifies the environmental performance of the port. The process has been based on educated, wellthought mechanisms based on the adoption of advanced IoT techniques and some mathematical help [15]. In particular, the technicalities of data acquisition and pre-processing have been designed decoupled from the indices' calculation. Whereas the former is distributed among diverse small pieces of processing (geographically dispersed or not) that follow the same structure and schemas, the latter is centralised within a PIXEL deployment 
acting as a model over the data to provide the target single metric figure. For obtaining the final value, some specific methods of normalisation, weighting and aggregation were selected out of several options after the analyses.

\subsection{A meaningful user experience}

A powerful tool like the PEI needs an equally powerful user interface, as a window to the full scope of the environmental efficiency and operations optimization at the port. The PIXEL dashboard is the module that allows to represent the data in a visual way, combine the data from multiple sources in a single view of truth and establish notifications to the relevant port staff, providing the right information on the right place at the right time. Dispersion of data sources and sensing inputs makes decision-making a tedious task hindered by the need of striving for reliable information. PIXEL is addressing this problem by integrating all data from multiple resources in a single view to offer better context and to drastically improve decision making. This is added by full configurability, a wide range of visualisations to envisage the data in the best way, using models and predictive algorithms, offering a unique place to show data or run a model to plan an activity or make an evidence-based business decision. It impacts the improvement of context awareness, better decision-making, and less time wasted looking for the appropriate data to analyse and visualise. It promotes better evaluation of environmental-related actions, helping prioritise and select the most impactful activities. The specific visualisations are made towards easy access of environmental performance and planning of port operations using alerts and existing models (see Fig. 3).

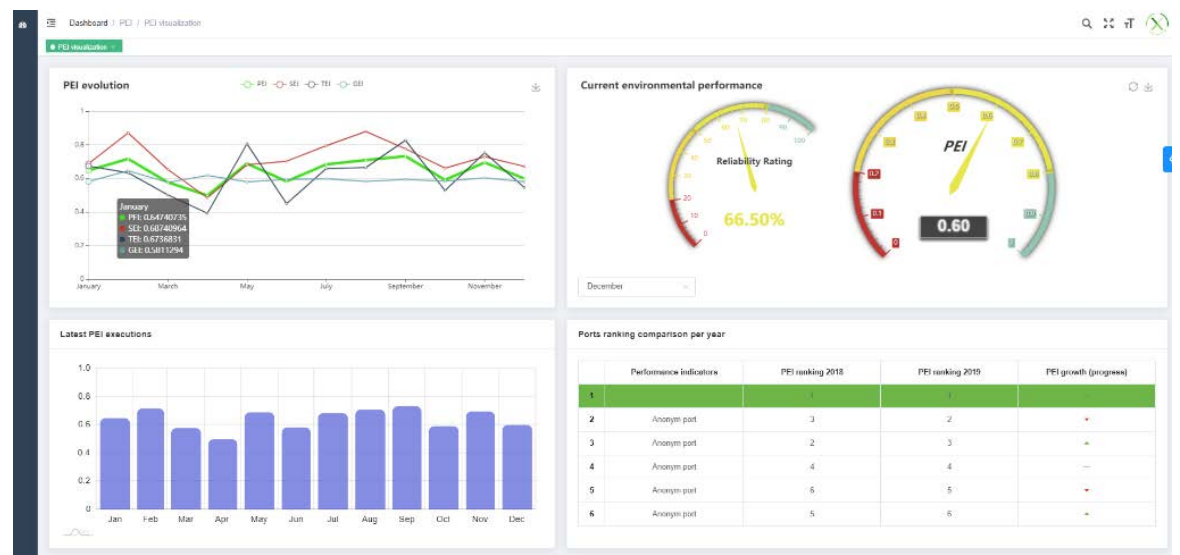

Figure 3: PIXEL Platform in action, through a meaningful interactive dashboard.

\section{EVIDENCE-BASED SIMULATION AT THE PORT}

The paradigm of the digital twin is not new although it has found in recent times the mature enough technological enablers to be able to flourish [16]. One of its most popular applications regards the empowerment of Industry 4.0 allowing for virtual models of physical (groups of) machines [17]. The approach is rapidly arriving to the maritime industry as well, following the digitalization of operations in ports and terminals, but also on the vessels at sea [18]. Though, due to the complex nature of port ecosystems, the digital twin concept does not transfer smoothly from the factory to the port (as noticed by the Port of Rotterdam, one of the pioneers in this area of research and development [19]). In what follows, we will be 
describing the contribution of PIXEL to this paradigm, and the benefits that follow from it in what relates to the planning of energy demand, environmental pollution management, and the management of the staff and infrastructure of the port to avoid contamination in the most recent COVID-19 context.

\subsection{The digitalisation of insightful information}

The digitalisation of the existing know-how at the port allows us to build a useful what-if scenario which can be seen as an important contribution to the digital twin of the port paradigm. It is an excellent example of the digital transformation of the maritime industry offering great benefits from the useful insights captured at the port management, already implemented and running in several European ports thanks to PIXEL. The Port Activity Scenario (called PAS) is an open-source system based on vessel calls and use of handling equipment specifications and supply chain (i.e., sequence of operations) in order to establish an operational description of the port activities related to cargo handling. Technically, the PAS uses a data-model listing all the considered activities' time series, feeding data to the PIXEL models in order for them to generate results. It is also a useful tool for planning activities at the port terminal, by providing forecasts based on past data e.g., vessels' cargo. It is adaptable in terms of data availability, i.e., it can work with a minimum set of data providing results of corresponding level of confidence. Moreover, it allows the manual reconfiguration of the schedule by the user and, once set up, allows each port to have a centralised repository of their resources.

The PAS tries to solve the problem of small and medium ports that might not have all the sensors installed yet, neither have a seamless way to centralise their information. The PAS provides data to the PIXEL models from the operations' schedule of the port. In the case of scarcity of sensors, the PAS can also provide data for the KPIs calculations of PEI, making it a central piece of the PIXEL architecture. Together with the energy model described in 3.2, it can provide useful insights (prediction, dispersion), on the energy consumption and production of a port (or an organisation in general).

Moreover, PAS predicts the kind of machinery that will be used and for how long in a terminal yard operating for a specific vessel, making use of the port calls, the machinery available and the chain of operations for each cargo (supply chain). The PAS simulates the operations of the terminal prioritising them and distributing the machinery operations across time and space (throughout the defined areas), based on the prioritisation of cargo, the supply chain that will be needed for the cargo and the availability of machinery. The user is also able to intervene and manually alter the prioritisations selected for each cargo as well as the machinery selection. Preliminary tests with PIXEL ports models for publishing, execution and scheduling show promising figures. Real tests in the different pilots with real models are ongoing, allowing simulation where energy and air pollution sensors are installed. Another innovation of PAS is rooting its execution on modularity, allowing it to generate new outcomes from the adaptation of general concepts to the maritime industry.

\subsection{Modelling energy (demand-supply) balance at the port}

For operational actors in ports, energy efficiency is an important issue from environmental and economic perspectives [20]. In time, ports would like to be able to optimise their energy flows. In this context, our aim is to model the port's energy demand and production to provide information about energy availability, reliability and efficiency. For doing so, a prerequisite is to quantify both energies consumption and production for ensuring balance in the context 
of complex industrial processes. With this model, a port will quantify the energy consumption corresponding to the data fed by the PAS, predict the local photovoltaic (PV) electricity production for a given period, and estimate the resulting electricity net balance between electricity consumption and production in the port area. This greatly improves the knowledge about energy consumption and production helping energy managers to better plan energy uses. It also allows ports to identify the type and quantity of energy used and help assess the related pollutants emission, driving decision-making on whether to integrate or not a PV system. Hence, it finds value in bringing better knowledge on how energy is used, being directly linked with cost savings.

For every considered cargo that is transiting inside the port, the corresponding sequence of operations across time is provided through the port activity scenario (PAS). This time series is the module's input. For each operation, the duration, the machine's energy type and its unit consumption values are available (from input and parameters combination). For each operation, the energy cost is calculated as the product of operation's duration and its machine's unit consumption. Note that the energy consumption independent of the PAS could also be considered and add to the energy's consumption time-series. Then for every energy's type, all the consumptions occurring during the same timeframe can be summed to get the corresponding total consumption. The sequence of all those time frames constitutes the energies consumption time-series. This technology, implemented in the Port of Bordeaux, is based on vessel calls and on the supply chain description deriving from PAS. It is easy to use and configure, requiring only the definition of the port's supply chains. Existing models of photovoltaic production will be integrated in new energy predictive algorithms of consumption in ports. Thus, renewable energy installations will be efficiently scaled, adaptive and new productive uses of over-produced energy can be developed. Its innovation lies in the complementarity between the energy production estimation and the energy consumption, which is a non-trivial question discussed in literature guidelines [21]-[23].

\subsection{Modelling environmental pollution}

By providing data about emissions quantification, the PAS allows exploration of pollution scenarios towards an always-greener port. In particular, it supports the association of emissions to the different types of modelled elements (trucks, machinery, areas, vessels, operations, etc.), either drawing from both "datasheet" specifications or inferring impact from their energy/fuel consumption. Port customers and community of stakeholders in the port environment (city, industries, vessels, all pollutant-emitting structures) might highly leverage such a feature to self-audit and understand the link between pollution levels (air, water, soil) and chemical composition and to investigate and assess the effects of pollution on the distribution of related health impacts, environments and socioeconomics in ports area and surroundings.

More complex simulations (based on physical equations) will allow them to assess highspatial resolution emissions/discharges, integrating modelling (different sectors/origins) on their business processes. This will cater for better assessment and quantification of environmental impacts (port activities including maritime traffic) for a better planning of environmental departments at the port. Monitoring of marine/air pollution in ports will put ports in a better position to diminish their associated environmental impact, leading to a reduction of air pollution in shipping activities within the port domain. For doing so, PIXEL has additionally developed a tool (highly based on AERMOD [24]) for simulating the air pollution dispersion in a port at a steady state. The model related considers direct and indirect consequences, in space and time, of the release of undesirable, dangerous or toxic substances. 
It simulates the dispersion of pollution plumes in each context and temporality, using mathematical tools and computer and mapping software. This is the first integrating system to generate global pollution indicators within few small European ports [25], generating data to refine results as (i) Air quality (combination of different sources dispersion of air pollutants); (ii) Noise (software including acoustic determinator); (iii) Water quality (combination of different sources dispersion of water pollutants).

Community uptake is expected to increase in the number of port users of this technology, and the multiplication of experienced feedback (integration of new sensors, new pollutants responding to the practices of port actors, etc). Optimisation of consumption and reduction of emission factors show that field data allows validation of models and simulations and allow for its scaling potential due to the extensive field of action, from open systems (coastal and offshore) to closed areas (bay, cove, ports, etc.) [26]. There is a growing demand from port customers and other stakeholders in their immediate environment impact (cities, industries, protected areas, etc.) [27].

\subsection{Addressing the impact of COVID-19 at the port}

The flexibility and adaptability of the PIXEL system enable the adoption of new models as new requirements arise in ports. Usually, the operations in a maritime port terminal (loading, unloading, moving cargo, in-yard works, etc.) imply the presence of many workers in the same area, arranged according to a tight schedule of processes that form a descriptive supply chain [28]. Social distancing measures (e.g., 1.5 or 2 meters between workers), although outdoors, have been mandatory in many of these installations during the last months.

Drawing from the needs expressed by ports [29], PIXEL has become an enabler to assist maritime ports monitor the impact of COVID-19 measures in this regard. By leveraging the Port Activity Scenario (PAS) model and other features of the platform, a tool has been developed to allow a port terminal to analyse that impact. In particular, the tool aims at informing how condensed (measured in number of workers per square meter) will each area in a terminal be in different moments of time depending on the vessels arriving at the port (see Fig. 4). The purpose of this service is to allow terminal managers to realise at which moment there would be a real risk of systematically not respecting social distancing and to

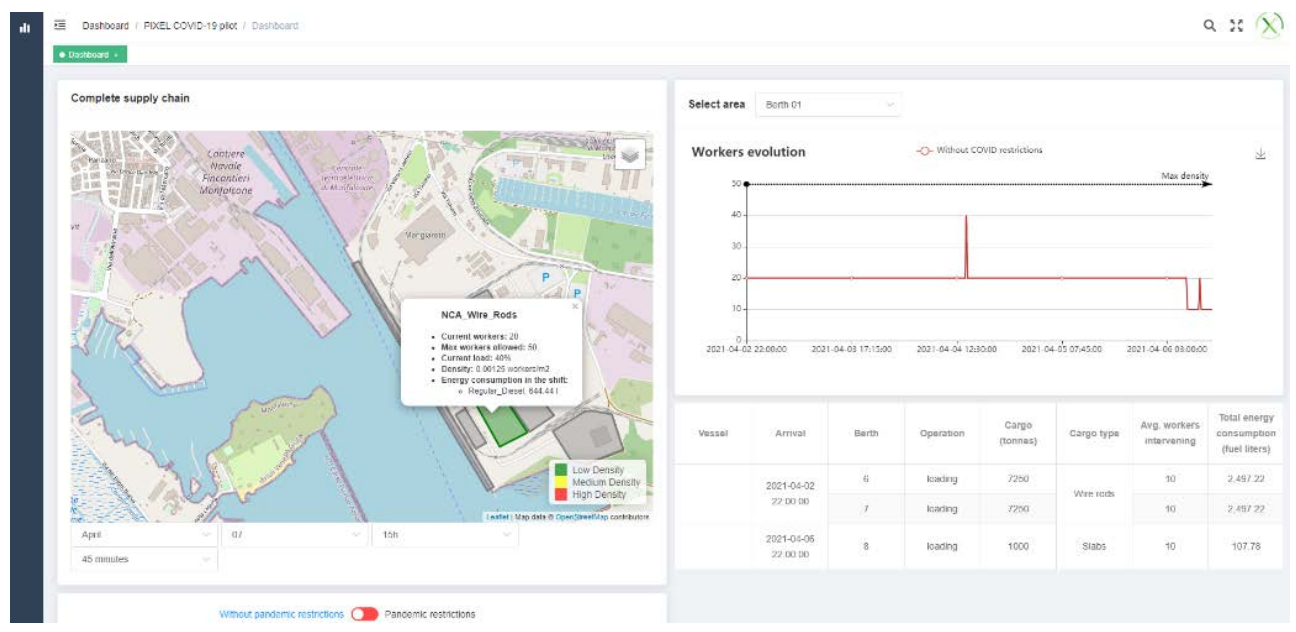

Figure 4: The COVID-19 pilot for port access availability. 
act thereupon. As a matter of fact, the tool is able to simulate an "alternative" schedule of operations considering that the established density thresholds (to be set up by the port) are not exceeded at any moment. For using such a model, one port should only be able to provide the vessel call list of forthcoming ships to berth at the docks and an actionable definition of their usual supply chains, machines, areas and cargo priorities.

\section{MARITIME DATA ANALYTICS}

With the fast digitalisation of the port industry, and the data collection from sensors in the context of the current approaches to the digital twin of the port, research problems within Big Data analytics arise, some of which are specific to the industry [30]. Moreover, powerful machine learning (ML) algorithms are not always useful or meaningful [31]. In this section we discuss these and the success stories from the angle of PIXEL's adoption.

\subsection{Artificial intelligence fit to the port}

The use of models and algorithms is not new in the ports environment. They are historically used to project Estimated Time of Arrival (ETA), to simulate traffic and dimension port infrastructure or to model processes with many variables to find optimal solutions (e.g., berth planning or work instruction assignment) [32], [33]. Other cases of algorithm-driven services in ports are the detection of maritime operations such mooring, berthing, anchoring, etc.

Thus, the use of Artificial Intelligence (AI) is not necessarily revolutionising how predictions, simulations, corrections or optimisations are done in maritime informatics [34]. However, the emergence of simple, accessible and less demanding narrow AI techniques [35] is a good opportunity to explore problems that in the past were complex to address and difficult to solve by using mathematical or modelling means. AI and particularly machine learning are typically indicated to solve problems that involve many inputs with non-defined relations among them or systems based on image recognition (especially when observation conditions are variable) [36]. To support this variety of algorithms of different nature, the architectural design of the platform must be adaptable to the specifics of each execution environment, yet to be capable of generalise the usage to ensure a smooth user experience. Here is where algorithm encapsulation (included in PIXEL) comes, to adapt to a heterogeneous spectrum of mathematical, process and AI algorithms and, at the same time, homogenise the interfaces to enable an accessible learning curve.

Consequently, one of the differentiating aspects of the PIXEL solution is that the algorithms are hidden from their technical implementation, as they are part of a port specific solution. The use of machine learning (ML) in PIXEL is a consequence of the user's needs and the chosen technique attends to effectiveness criteria, considering the different ways to solve a problem, the resources demanded and the impact of the problem in the port business. The application of artificial intelligence in the platform is, therefore, driven by user experience and business needs and is not a pre-defined technological choice leading to unnecessary complexity: right algorithms are chosen to the right problems, avoiding excess of parameterisation selection of approaches or configuration, as can be seen in more generic solutions (as we see for instance in the commercial offer of BigML).

\subsection{Building business intelligence from data}

PIXEL aims to provide useful insights from the port data obtained from the digitalisation of ports. PIXEL answers that question by providing powerful algorithms feeding on different types of data sources (Automatic Identification System (AIS) - FAL forms [37] - Satellite) 
that improve the business intelligence at the port, enhancing ETA/ETD and other optimizations of vessel traffic and manoeuvring with ML methods aiming for port digitalisation. These PIXEL tools help discover patterns such as seasonality, regularity, etc. that impact port businesses. Moreover, those allow for further insight on the operations held in the overall maritime ecosystem around the port, and improves the business intelligence at the port based on optimized openly accessible AIS data. Particularly, this improves ETA/ETD, helping to plan arrival/departure times to minimise congestion at the port, optimising costs/gains. Furthermore, it identifies unusual behaviour in the port area, improving port/shipping operations. Ports have access to FAL forms and AIS data (untrusted due to known $30 \%$ inaccuracy), but are not efficiently using it to build business intelligence. PIXEL tackled this problem with a set of AI-based algorithms capable of predicting turnaround time from FAL forms, and to predict vessel short-term ETA based on AIS data. Most of the AIS-based algorithms were designed to compete globally with state-of-the-art (SotA) research, applying SotA image recognition algorithms and methods to the context of the maritime industry, aligning to the specific challenges in ports [38].

\subsection{Intermodal transportation forecasting}

Intermodal transport is an essential part of logistics and has a fundamental role in PIXEL over three different main modes of transportation use: urban road, highway and train. PIXEL offers an innovative multimodal transport solution based on advanced models of cooperation with the port and predictive algorithms aiming to avoid congestion. The technology allows to compare economic and environmental time impacts of different transport modes, supporting the planning of port transport operations by monitoring the environmental impacts, while endorsing better freight transport in-out of port with business operators and other regional nodes for reducing the impact on hinterland and environment.

The problem faced by ports and logistics operators is how to improve the capacity of planning [39]. This is addressed in PIXEL with technology to support the optimization of the port resources, in particular of the port area, through a more accurate planning of the traffic in-out of the port. The developed tool supports the planning of port transport operations monitoring the environmental impacts, and can support a shared planning of freight transport with business operators in order to reduce the impact on hinterland and environment. It is based on two models related with transport: an "intermodality sub model" related to intermodality way of transport applied on slabs traffic between the Port of Monfalcone and Aussa Corno industrial district; and a "traffic sub model" related to traffic and parking inside port premises and the possible rerouting to the interport of Gorizia.

Additionally, PIXEL provides a set of AI-based algorithms capable of predicting traffic congestion at the port. It includes the detection of navigation and port operations using predictive AI-based algorithms using available AIS data. The model is merging existing technologies and existing data to provide a new service to the users, complemented by the novel predictive algorithms to forecast road congestion. It allows ports to compare economic and environmental time impacts of different transport mode, while supporting the planning of port transport operations monitoring the environmental impacts.

\section{INSIDE THE PIXEL ENGINE}

In what follows we shall focus on the Big Data and IoT infrastructure underneath the PIXEL platform that was built following a lean product development manner [40], attending to the needs and requirements of the pilot ports and the reality of their data. 


\subsection{Secure data acquisition with FIWARE agents}

The port digitalisation holds a big role in data technologies and IoT, but also has great expectations that are sometimes hard to satisfy due to the maturity of available technologies [41]. For that reason, PIXEL makes available plug-and-play functionality to connect the port data to the system. This allows for the adoption of a large variety of sensing options and data types that can better fit the resources and budget of the port, allowing for ports of all sizes to collect the needed data feeding the computations in PEI and PAS. PIXEL makes available a series of data acquisition agents ready to serve, but others can be created by the user with the help of a generic development framework built for that aim.

The Data Acquisition Layer is a FIWARE-based system that enables external data sources to interface with the PIXEL Information Hub (described in Section 5.2) and to convert any data format to PIXEL Data Models. The Data Acquisition allows users to push data to the Orion broker [5], which is used by the Information Hub to subscribe to data events. Orion and the subscription API - Application Programming Interface - are FIWARE standards, available and documented on the literature. Data Agents are easily managed through an orchestrator and easy to develop using a dedicated framework [4].

Data is critical for ports; the challenge is to be able to integrate multiple data sources, heterogeneous and presenting potential security risks. The data acquisition layer in PIXEL is an assembly of software components that allow FIWARE-based data authentication, acquisition and ease of integrating new data sources. The technology ensures data ingestion, data cleaning and data validation, using FIWARE open-source standards, allowing structuring data to be used intelligently. It eases the connection of new data sources. In that, IoT data sources and port communication system data are easily integrated into PIXEL. Moreover, it ensures data source authentication via strong security mechanisms.

\subsection{Big Data information hub}

As data fed from, e.g., sensing at the port, is arriving in rapidly growing amounts with different frequency and complexity, the data infrastructure of yesterday (if at all) is not enough [42]. The port of the future needs a Big Data engine that is flexible and resourceful, that enables us to get insights from that data and make it useful. PIXEL provides the port with the Information Hub (IH), a Big data engine fitting the most demanding data needs of all stakeholders in the maritime industry, modular and flexible, ready for third-party integration.

It can leverage connectivity, with support for FIWARE data models conversion, data sources discovery and data sources integration, and supports high-volume and high-quality time series data acquisition, being easily integrable with several PCS systems. Moreover, it can be triggered by timing events or on-change, reducing over time or aggregation of historical data on a configurable basis. It allows the user to query, filter, and display gathered data, and manage near real-time or archived measurements.

Furthermore, it is an enabler for high frequency and high volume data; well defined open interface to connect different data sources at the port. It is easy to integrate with existing solutions via API. It is scalable, allowing to plug in different modules/tools to have real-time predictions, offline non real time model processing, and single point to find data.

The IH smoothly integrates high frequency Big Data sources and makes the data easily available. It is based on technology built to support particle acceleration technologies, using Elasticsearch, Apache Kafka, etc. The data is inserted into the system through different realtime stream sources, and it is prepared to be sourced over the API. It is a centrepiece of 
PIXEL, that allows storing data on a configurable base of resolution in time, triggered by timing events or on-change. Data may be either gathered from devices, higher level data like computed properties or even abstract-generated. Base data manageability features are not exposed to the port management staff (end users), but only to specialised ICT personnel in charge of data management can vouch for this solution. A good Big Data management will be essential for the digitalisation of the port, with IoT and sensor data collection. Only with Big Data infrastructure fit to the needs of the port of the future that the big step of industrial digitalisation can be taken.

\subsection{Computer model management}

Controlling and managing models and algorithms to run upon port data becomes a cornerstone for letting ports address the needs of tomorrow. PIXEL provides its own operational tools (OTs) as a natural framework to manage the publication and execution of models and predictive algorithms. Therefore, models and predictive algorithms are represented in a standard way and can be managed properly, in a similar way as WSDL (Web Services Description Language) describes web services and the way they can be invoked. It allows for new models to be integrated into the system, providing PIXEL with an additional sense of modularity. It also allows defining FIWARE-compliant KPIs to be monitored.

These address the difficulty of integrating different models in an enterprise platform due to its natural heterogeneity. They have a standard format to describe services, based on the invocation syntax aligned with standard description, ensuring independence through private or public Docker instances with self-management in their own premises and offering REST API management. The OTs define a standard JSON format for publication and execution of models and predictive algorithms and manage the execution through Docker containers. They manage services (models and predictive algorithms) in an automated and isolated way through Docker containers, facilitating cloud usage. Moreover, they describe and manage different models and predictive algorithms with different inputs and implementation approaches. It is also able to be executed by OTs sporadically or in a scheduled way.

To sum up, the OTs framework is a middleware to manage dockerized models and predictive algorithms, consisting of a custom, open-source tool able to handle PIXEL compliant models and predictive algorithms. The OTs allow adding new functionalities if PIXEL grows and new demands from stakeholders are requested It is a powerful publication and execution tool for models and predictive algorithms that is built to ensure modularity in a new perspective: over the computational models it includes.

\section{CONCLUSIONS AND RECOMMENDATIONS}

The concept of smart port highly relies on using smart and green technologies to increase a port's efficiency, improve performance, innovation, flexibility, environmental safety and economic competitiveness. Developing such a port represents a significant progress in the application of energy conservation and emission reduction in the context of the application of state-of-the-art technologies in ports and maritime shipping sectors. It makes use of powerful interpretive structural modelling analysis to be able to divide the factors into different levels and to draw an innovative approach for green and smart port construction structures [43]. The PIXEL project has provided a series of results (outlined in this paper) that show the value of a smart-port-of-the-future initiative towards an all-encompassing technological governance framework of a green port. The achievements of PIXEL provide a practical tool, a decision-making basis and information reference for international 
organisations, relevant governments or policymakers to formulate reasonable and effective governance strategies for green and smart port development. For a greater improvement of environmental sustainability and efficiency, ports should attach greater importance to cooperation and development, strengthen cooperation between them and their associated stakeholders, industries and shipping departments, and jointly promote the concept of green and smart port development. The concept of sustainable development should be also boosted. Integrated development contributes to enhancing port competitiveness as well as to accommodating issues of sustainability with artificial intelligence, and Big Data environment. Such smart technologies, such as Big Data, Internet of Things (IoT), Artificial Intelligence (AI) and other forms of smart technology methods will improve performance and economic competitiveness and environmental sustainability of the ports.

\section{ACKNOWLEDGEMENT}

PIXEL project has received funding from the European Union's Horizon 2020 research and innovation programme under grant agreement $\mathrm{N}^{\circ} 769355$.

\section{REFERENCES}

[1] PortTechnology, https://porttechnology.org/news/port_of_rotterdam_creating_global _logistics_chains/. Accessed on: Apr. 2021.

[2] Hofmann, W. \& Branding, F., Implementation of an IoT-and cloud-based digital twin for real-time decision support in port operations. IFAC-PapersOnLine, 52(13), pp. 2104-2109, 2019.

[3] Taylor, N., Human, C., Kruger, K., Bekker, A. \& Basson, A., Comparison of digital twin development in manufacturing and maritime domains. International Workshop on Service Orientation in Holonic and Multi-Agent Manufacturing, Springer, pp. 158170, 2019.

[4] Simon, E., Garnier, C., Lacalle, I., Costa J.P. \& Salvador, C.E.P., Small and medium ports' activities: Introduction to the PIXEL approach. WIT Transactions on the Built Environment, vol. 187, WIT Press: Southampton and Boston, pp. 149-163, 2019.

[5] FIWARE ORION, https://fiware-orion.readthedocs.io/en/master/. Accessed on: Mar. 2021.

[6] Martinčič, T., Štepec, D., Costa, J., Čagran, K. \& Chaldeakis, A., Vessel and port efficiency metrics through validated AIS data. Proceedings of OCEANS 2020 MTS/IEEE, 2021.

[7] European Commission, A European Green Deal, 2020. https://ec.europa.eu/info/ strategy/priorities-2019-2024/european-green-deal_en. Accessed on: 9 Mar. 2021.

[8] Zheng, Y. et al., Port city sustainability: A review of its research trends. MDPI Sustainability, 12, p. 8355, 2020. DOI: 10.3390/su12208355.

[9] PIXEL, D5.4 PEI manual for adoption in ports and guidelines for WP5 environment and society. European Commission, 2021.

[10] European Commission, Trans-European Transport Network (TEN-T), 2019. https://ec.europa.eu/transport/themes/infrastructure/ten-t_en. Accessed on: 9 Mar. 2021.

[11] DocksTheFuture, D1.6 port of the future concepts, topics and projects-consolidated versions, 2021. https://www.docksthefuture.eu/results/. Accessed on: 9 Mar. 2021.

[12] Flam Port, Environmental port index, 2021. https://www.flamport.no/environmentalport-index-epi. Accessed on: 9 Mar. 2021.

[13] WPSP, Environmental ship index, 2021. https://www.environmentalshipindex.org/. Accessed on: 9 Mar. 2021. 
[14] Široka, M., Piličić, S., Milošević, T., Lacalle, I. \& Traven, L., A novel approach for assessing the ports' environmental impacts in real time - The IoT based port environmental index. Ecological Indicators, 120, p. 106949, 2021.

[15] PIXEL, D5.3 PEI definition and WP5 algorithms v2. European Commission, 2020.

[16] Boyles, R., How the port of Rotterdam is using IBM digital twin technology to transform itself from the biggest to the smartest. https://www.ibm.com/blogs/internetof-things/iot-digital-twin-rotterdam/. Accessed on: 9 Mar. 2021.

[17] Cinar, Z., Nuhu, A., Zeeshan, Q. \& Korhan, O., Digital twins for industry 4.0: A review. 2020. DOI: 10.1007/978-3-030-42416-9_18.

[18] Smart Maritime Network. https://smartmaritimenetwork.com/2020/07/15/digitaltwins-for-the-maritime-sector/. Accessed on: Apr. 2021.

[19] E. Rademaker, The digital twin of port of Rotterdam. https://youtu.be/nUbgHGJXj5c. Accessed on: 9 Mar. 2021.

[20] Sdoukopoulos, E., Boile, M., Tromaras, A. \& Anastasiadis, N., Energy efficiency in European ports: State-of-practice and insights on the way forward. MDPI Sustainability, 11, p. 4952, 2019. DOI: 10.3390/su11184952.

[21] Iris, C. \& Lam, J., A review of energy efficiency in ports: Operational strategies, technologies and energy management systems. Renewable and Sustainable Energy Reviews, 112, pp. 170-182, 2019. DOI: 10.1016/j.rser.2019.04.069.

[22] European Commission. https://ec.europa.eu/eurostat/documents/38154/4956218/ ENERGY-BALANCE-GUIDE-DRAFT-31JANUARY2019.pdf/cf121393-919f4b84-9059-cdf0f69ec045. Accessed on: Apr. 2021.

[23] Hellenic Shipping News. https://www.hellenicshippingnews.com/offshore-energygoes-green-part-1-energy-balance-simulations/. Accessed on: Apr. 2021.

[24] Rood, A.S., Performance evaluation of AERMOD, CALPUFF, and legacy air dispersion models using the Winter Validation Tracer Study dataset. Atmospheric Environment, 89, pp. 707-720, 2014.

[25] PIXEL project. https://pixel-ports.eu/wp-content/uploads/2019/02/D4.1_PIXELModels-v1.pdf. Accessed on: Apr. 2021.

[26] Baawain Mahad, S. \& Al-Serihi Aisha, S., Systematic approach for the prediction of ground-level air pollution (around an industrial port) using an artificial neural network. Aerosol and Air Quality Research, 14, pp. 124-134, 2014.

DOI: 10.4209/Aaqr.2013.06.0191.

[27] Mocerino, L. et al., A methodology for the design of an effective air quality monitoring network in port areas. Scientific Reports, 10, p. 300, 2020.

DOI: $10.1038 / \mathrm{s} 41598-019-57244-7$.

[28] United Nations, COVID-19 and maritime transport: Impact and responses, 2020. https://unctad.org/system/files/official-document/dtltlbinf2020d1_en.pdf. Accessed on: Mar. 2021.

[29] PortForward, How Ports de Balears use case "Monitoring of Port Authority's activities" could help to minimize the effect of COVID-19, 2021. https://www.portforward-project.eu/how-ports-de-balears-use-case-monitoring-ofport-authoritys-activities-could-help-to-minimize-the-effect-of-covid-19/. Accessed on: 9 Mar. 2021.

[30] Munim, Z., Dushenko, M., Jaramillo, V., Shakil, M. \& Imset M., Big data and artificial intelligence in the maritime industry: A bibliometric review and future research directions. Maritime Policy \& Management, 47(5), pp. 577-597, 2020.

DOI: $10.1080 / 03088839.2020 .1788731$. 
[31] Anolytics. https://www.anolytics.ai/blog/how-to-select-suitable-machine-learningalgorithm-for-a-problem-statement/. Accessed on: Apr. 2021.

[32] University of Twente. http://essay.utwente.nl/82201/1/Flapper_BA_EEMCS.pdf. Accessed on: Apr. 2021.

[33] Amazon AWS. https://aws.amazon.com/blogs/machine-learning/using-machinelearning-to-predict-vessel-time-of-arrival-with-amazon-sagemaker/. Accessed on: Apr. 2021.

[34] Lind M. et al., Maritime Informatics, Springer, 2020.

[35] Stripped Giraffe. https://www.striped-giraffe.com/en/lightweight-machine-learningand-analytics/. Accessed on: Apr. 2021.

[36] Dev.to. https://dev.to/piyushbagani15/neural-networks-use-cases-in-the-real-world2dk1. Accessed on: Apr. 2021.

[37] IMO, FAL forms and certificates, 2021. https://www.imo.org/en/OurWork/ Facilitation/Pages/FormsCartificates-default.aspx. Accessed on: 9 Mar. 2021.

[38] Štepec, D., Martinčič, T., Klein F. \& Costa, D.V.a.J.P., Machine learning based system for vessel turnaround time prediction. Proceedings of the 21st IEEE International Conference on Mobile Data Management (MDM), pp. 258-263, 2020.

[39] Oudani, M., Benghalia, A., Boukachour, J., Boudebous, D. \& Ahmed A., Innovative port logistics through coupled optimization/simulation approaches. 2018. DOI: 10.4018/978-1-5225-5273-4.ch013.

[40] Pessôa M. \& Trabasso, L., The Lean Product Design and Development Journey, Springer International Publishing, 2017.

[41] Kapidani, N., Bauk, S. \& Davidson, I., Digitalization in developing maritime business environments towards ensuring sustainability. MDPI Sustainability, 12, p. 9235, 2020. DOI: $10.3390 / \mathrm{su} 12219235$.

[42] Mindef. https://www.mindef.gov.sg/oms/safti/pointer/documents/pdf/monthlyissue/ sep2020.pdf. Accessed on: Apr. 2021.

[43] Chen, J., Huang, T., Xie, X., Lee, P.T.W. \& Hua, C., Constructing governance framework of a green and smart port. Journal of Marine Science and Engineering, 7(4), p. 83, 2019. 